\title{
NKG2A silencing in effector cells to improve effectivity of cell based therapeutics
}

\author{
C Figueiredo*, J Zenk, B Eiz-Vesper, R Blasczyk and A Seltsam
}

Address: Institute for Transfusion Medicine, Hannover Medical School, Hannover, Germany

* Corresponding author

from 12th Joint Meeting of the Signal Transduction Society (STS). Signal Transduction: Receptors, Mediators and Genes Weimar, Germany. 29-3I October 2008

Published: 26 February 2009

Cell Communication and Signaling 2009, 7(Suppl I):A29 doi:10.1I86/1478-8IIX-7-SI-A29

This abstract is available from: http://www.biosignaling.com/content/7/SI/A29

(c) 2009 Figueiredo et al; licensee BioMed Central Ltd.

The heterodimeric NKG2A/CD94 receptor delivers an inhibitory signal upon recognition of HLA-E molecules. In several studies it has been demonstrated that signalling via NKG2A/CD94 receptor significantly reduces T or NK cell cytotoxic activity and cytokine production. In hematopoietic stem cell transplantation, expression of NKG2A on NK and T cells has been shown to compromise the graft-versus-leukemia effect. In addition, NKG2A was shown to inhibit tumor-specific $\mathrm{T}$ cell responses. In this study, we developed a RNAi-based approach to permanently silence the expression of NKG2A molecules on NK and T-cells. The functional relevance of NKG2A silencing for the cytotoxic potential of genetically engineered NK and T-cells was evaluated.

$\mathrm{NKG}_{2} \mathrm{~A}^{+}$cells were isolated from fresh PBMCs. Lentiviral vectors were designed to express short hairpin RNA sequences (shRNA) targeting NKG2A transcripts. The level of NKG2A suppression was measured by flow cytometry and real-time RT-PCR. The effect of NKG2A receptor silencing on the cytolytic potential of $\mathrm{NK}$ and $\mathrm{T}$ cells was evaluated in cytotoxicity assays using K562 and B-LCL cells as targets. In addition, granzyme B mRNA transcript levels were detected by real-time RT-PCR.

The transduction of inducible RNAi cassettes containing the sequences for shRNAs targeting NKG2A caused a reduction of protein expression by up to $80 \%$ in NK and $\mathrm{T}$ cells. In cytotoxicity assays, it was demonstrated that NKG2A silencing was effective to enhance $\mathrm{NK}$ and $\mathrm{CD} 8^{+} \mathrm{T}$ cell lysis by up to $40 \%$. In comparison with unmodified cells, granzyme B transcript levels were upregulated by up to 12-fold in NKG2A silenced cells after target exposure. Expression of NKG2A-specific shRNA did not affect the expression of the activating markers NKp44 on NK cells and CD25 on T cells.

Our data suggest that RNAi-mediated silencing of NKG2A in effector cells could improve the effectivity of cell-based immunotherapeutics and might be of particular interest in an autologous approach. 